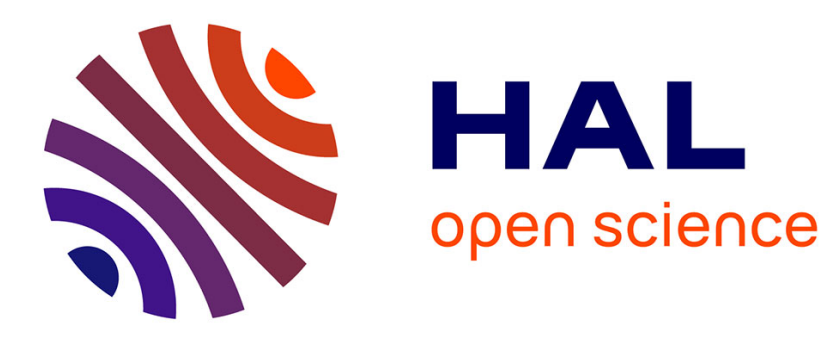

\title{
Time-resolved photoelectron and photoion fragmentation spectroscopy study of 9-methyladenine and its hydrates: a contribution to the understanding of the ultrafast radiationless decay of excited DNA bases. C. Canuel, Mohamed Elhanine, M. Mons, F. Piuzzi, B. Tardivel, I. Dimicoli
}

\section{- To cite this version:}

C. Canuel, Mohamed Elhanine, M. Mons, F. Piuzzi, B. Tardivel, et al.. Time-resolved photoelectron and photoion fragmentation spectroscopy study of 9-methyladenine and its hydrates: a contribution to the understanding of the ultrafast radiationless decay of excited DNA bases.. Physical Chemistry Chemical Physics, 2006, 8, pp.3978-3987. 10.1039/b606437j . hal-00091945

\section{HAL Id: hal-00091945 \\ https://hal.science/hal-00091945}

Submitted on 7 Sep 2006

HAL is a multi-disciplinary open access archive for the deposit and dissemination of scientific research documents, whether they are published or not. The documents may come from teaching and research institutions in France or abroad, or from public or private research centers.
L'archive ouverte pluridisciplinaire HAL, est destinée au dépôt et à la diffusion de documents scientifiques de niveau recherche, publiés ou non, émanant des établissements d'enseignement et de recherche français ou étrangers, des laboratoires publics ou privés. 
Time-resolved photoelectron and photoion fragmentation spectroscopy study of 9-methyladenine and its hydrates: a contribution to the understanding of the ultrafast radiationless decay of excited DNA bases.

Clélia Canuel*, Mohamed Elhanine, Michel Mons, François Piuzzi, Benjamin Tardivel and Iliana Dimicoli.

Laboratoire Francis Perrin (URA 2453 CEA-CNRS), CEA Saclay, Bat. 522, 91191 Gif-surYvette Cedex, France

\section{Abstract}

The excited state dynamics of the purine base 9-methyladenine (9-MeAde) has been investigated by time- and energy-resolved photoelectron imaging spectroscopy and massselected ion spectroscopy both in vacuum and water-cluster environment. The specific probe processes used, namely a careful monitoring of time-resolved photoelectron energy distributions and of photoion fragmentation, together with the excellent temporal resolution achieved enable us to derive additional information on the nature of the excited $\left(\pi \pi^{*}, \mathrm{n} \pi^{*}\right.$, $\pi \sigma^{*}$, triplet) states involved in the electronic relaxation of adenine. The two-step pathway we propose to account for the double exponential decay observed agrees well with the recent theoretical calculations. The near UV photophysics of 9MeAde is dominated by the direct excitation of the $\pi \pi^{*}\left({ }^{1} \mathrm{~L}_{\mathrm{b}}\right)$ state (lifetime of $100 \mathrm{fs}$ ), followed by internal conversion, proceeding to the $n \pi^{*}$ state (lifetime in the ps range) via conical intersection. No evidence for the involvement of a $\pi \sigma^{*}$ or a triplet state was found. 9MeAde-water clusters have been studied, focussing onto the fragmentation of these species after the probe process. A careful analysis of the fragments allowed us to provide evidence for a double exponential decay profile for the hydrates. The very weak second component observed, however, leads to conclude to a very different photophysics as compared to the isolated base, assigned to a competition between i) a direct one-step decay of the initially excited state $\left(\pi \pi^{*} L_{a}\right.$ and $/$ or $L_{b}$, stabilised by hydration) to the ground state and ii) a modified two-step decay scheme, qualitatively comparable to that occurring in the isolated molecule.

\footnotetext{
* corresponding author; e-mail address : clelia.canuel@cea.fr
} 


\section{Introduction}

The photodamage of DNA continues to be a subject of intense interest to biologists, chemists and physicists. DNA bases are the only nucleic acid components that can be electronically excited by the sun's ultraviolet radiation. Therefore the study of the conversion pathways of the substantial energy deposited by light absorption is of great importance. ${ }^{1-4}$ The excited $\pi \pi^{*}$ states of these complex aromatic systems mostly undergo non-radiative relaxation, namely either internal conversion or intersystem crossing to other dark or weakly allowed states, which could initiate photochemical processes. It is often stated that these latter processes are made highly improbable by a very fast (picosecond) internal conversion to the electronic ground state where the electronic energy is eventually thermalised, providing nucleobases with a high level of photostability. ${ }^{5-8}$ However, the detailed mechanism governing the excited state dynamics of the bases is far for being fully understood. In particular, the branching ratio between reactive and non reactive relaxation pathways specifically for each DNA base is not established and needs further investigations.

The present work is devoted to one of the most studied species, the purine base adenine (9H-Ade canonical isomer and its related methylated compound 9MeAde) for which we can hope to collect robust experimental data in order to address this fundamental issue. Recent advances in time-resolved spectroscopic studies, both in the liquid and gas phase, and considerable theoretical efforts have lately brought some insight on the nature and the properties of the lowest electronically excited states of this base ${ }^{6}$. We shall begin with a short review of these recent results.

The electronic spectrum of the canonical form $9 \mathrm{H}$-Ade is rather complex and consists of several closely spaced $\pi \pi^{*}, \mathrm{n} \pi^{*}$ and $\pi \sigma^{*}$ states. Theoretically, three low-lying singlet excited states have been found in the isolated base, giving rise to two electronic transitions of $\pi \pi^{*}$ character $\left(\mathrm{L}_{b}\right.$ in the near UV and $\mathrm{L}_{\mathrm{a}}$ at higher energy), and a very weak one of $n \pi^{*}$ character. $^{9-13}$ The $L_{a}$ state is the most strongly allowed state in the absorption spectrum. At higher energies, Rydberg states characterised by highly diffuse $\sigma$ orbitals, localized mostly on the azine and amino groups, have also been predicted. ${ }^{13-16}$ The molecular beam UV spectrum of 9H-Ade (and similarly for 9MeAde) is composed of a small number of resolved vibronic bands followed by an unstructured absorption at higher energy, which coincides with a fluorescence break-off. ${ }^{17-21}$ Experimentally, both the frequency- and time-resolved observations in the gas phase indicate the existence of a rapid excited-state relaxation process 
with a low barrier $\left(\sim 800 \mathrm{~cm}^{-1}\right) .{ }^{17-27}$ The excited states dynamics exhibit a strong dependence on the excitation energy, with characteristic lifetimes ranging from a few tens of picoseconds near the origin to about one picosecond at excess energy of only $\sim 1300 \mathrm{~cm}^{-1} .^{18,22-27}$ At this energy $(267 \mathrm{~nm})$, the excited molecule (9MeAde or 9HAde) decays mainly following a twostep mechanism with a short and long component of about $110 / 1300 \mathrm{fs}^{27}$ assigned to the lifetimes of the optically bright $\pi \pi^{*} \mathrm{~L}_{\mathrm{b}}$ and the weakly allowed $n \pi^{*}$ states, respectively. At higher excitation energies $(250 \mathrm{~nm})$ a similar temporal behaviour has been observed ${ }^{24,28}$ as measured by both photoionisation and photoelectron spectroscopy. The localization of the excitation on the six-member ring and the key role of the different vibrational modes (ring buckling, amino group inversion) in the relaxation process have been evidenced by the dynamical behaviour of different methyl-substituted adenines and 2-aminopurine (isomer of adenine $)^{27}$. Other studies on $\mathrm{Ade}^{25}$, Ade-water clusters ${ }^{28}$ and Ade in water solution ${ }^{29}$ have suggested that $\pi \sigma^{*}$ states might play an important role in the excited state relaxation. In room temperature aqueous solution, subpicosecond lifetimes, found to be not dependent on the excitation energy, have been observed. After excitation at $267 \mathrm{~nm}$, the decay is monoexponential for 9MeAde $(0.22 \mathrm{ps})$ as observed by transient absorption $^{6}$ but monoexponential or biexponential as observed by fluorescence up-conversion for the ribonucleoside adenosine $(\sim 0.31 \mathrm{ps})^{29}$ and the 2 '-deoxyadenosine (dAdo) $\sim 100 / 420$ fs or 2'deoxyadenosine monophosphate (dAMP) $\sim 100 / 520 \mathrm{fs}$, respectively ${ }^{30}$. Early time-resolved studies on adenine-water clusters reported on monoexponential decays $(\sim 60-220 \mathrm{fs})^{28,31}$.

On the theoretical side, several mechanisms were proposed to account for the ultrafast deactivation ${ }^{14-16,32-35}$. Most of them involve a coupling between the optically bright and therefore preferentially photo-excited $\pi \pi^{*}$ state and the $n \pi *$ state. Reactive and non reactive relaxation channels have been found theoretically. They proceed via conical intersections (CI) of the respective potential energy surfaces (PES), whose accessibility is governed by barriers of various heights. The N9-H loss or the opening of the five-member ring involving Rydberg states, ${ }^{13,14}$ for example, are predicted to happen at fairly high energy $(\sim 200 \mathrm{~nm}$ and $230 \mathrm{~nm}$, respectively) as compared to the almost barrierless $(\sim 0.1 \mathrm{eV})$ internal conversion to the ground state which opens near the absorption threshold. ${ }^{14}$

This paper intends to bring together new experimental results as well as critically reviewed previously published material in order to provide a consistent set of data on the adenine system and its water clusters, to be compared with the theoretically proposed relaxation mechanisms. Among the low-energy excited states $\left(\mathrm{L}_{\mathrm{b}}, \mathrm{L}_{\mathrm{a}}, \pi \sigma^{*}, \mathrm{n} \pi^{*}\right)$, the role of 
the state with the strongest oscillator $\mathrm{L}_{\mathrm{a}}$ has not been considered. The contribution of this state to the dynamics involving the $\mathrm{L}_{\mathrm{b}}$ and $\mathrm{n} \pi^{*}$, and the branching ratio between these states in the dynamics of hydrogen bonded adenines (water solution or Ade-water clusters) need further investigation. With these premises, considerable importance is attributed to precise experiments on the bases, in particular the biologically relevant species 9Me-Ade and its water solvated species. Substitution of the N9 hydrogen atom by a methyl group blocks tautomerisation, thus eliminating the presence of $7 \mathrm{H}-\mathrm{Ade}$, and suppresses a non relevant hydration site. It then provides a reasonable model for the base moiety in Ado, dAdo and dAMP, allowing a meaningful comparison with aqueous results on these species. ${ }^{6}$

Here we report the first results obtained by time-resolved velocity map photoelectron imaging spectroscopy on 9MeAde. The mass selective photoionisation diagnosis carried out in parallel guarantees the origin of the observed signals. In addition, the time-resolved measurements of fragmented species are shown to provide valuable information on the vibrational content of the system ${ }^{36,37}$. These complementary techniques provide a consistent set of data allowing us to determine the nature of the electronic excited states, in agreement with the theoretical approaches. Then, in an attempt to bridge the gap between isolated molecules and biological environment, the photophysics of the model nucleoside 9MeAde in water clusters has been investigated by time-resolved photoionisation spectroscopy, with special attention given to the fragmentation process.

\section{Experimental}

The experimental setup combines a pulsed supersonic jet with femtosecond pulsed lasers and a detection system that can analyze alternatively photoion signals through a timeof-flight mass spectrometer (TOF-MS) or photoelectron signals through a velocity map imaging device.

The beam is generated from a coexpansion of the carrier gas (argon or helium) with nucleobases produced in a temperature-controlled oven placed upstream the pulsed valve (General Valve). Typical operating temperatures were about $140^{\circ} \mathrm{C}$. Commercial products from Aldrich were used without further purification. In order to form bases hydrates, the carrier gas was seeded by passing through an upstream temperature-controlled water reservoir. The stagnation pressure $\mathrm{P}_{0}(\sim 1-2$ bars $)$ and the temperature of the water reservoir $\left(-10^{\circ} \mathrm{C}\right)$ were kept low to avoid water aggregation and large distribution of adenine-water clusters. 
The LUCA laser facility at Saclay delivers the second $(400 \mathrm{~nm})$ and third (267 nm) harmonics of the output of a Ti:Sapphire regenerative amplifier operating around $800 \mathrm{~nm}$ at a $20 \mathrm{~Hz}$ repetition rate. The two nearly collinear pump and probe laser beams can be focused independently onto the molecular beam. The power density of the probe laser was kept lower than $2 \times 10^{11} \mathrm{~W} . \mathrm{cm}^{-2}$, which is enough to perform multiphoton ionization of the electronically excited adenine. For all measurements, pump (267 nm) and probe (400 or $800 \mathrm{~nm}$ ) laser energies and their focusing conditions were carefully adjusted to minimize one-colour ionization. The IR fundamental light $(800 \mathrm{~nm})$ was eliminated when using the $400 \mathrm{~nm}$ probe beam by successive reflections on interferometric multilayer mirrors. The fundamental or second harmonic output of the probe laser goes through a computer-controlled delay line, allowing us to vary the time delay relative to the $267 \mathrm{~nm}$ pump laser pulse over about $600 \mathrm{ps}$. The temporal profiles were acquired either by randomly setting the pump-probe delays from a predetermined list of values (for ranges of a few ps) or by scanning the range back and forth and averaging (for decays of several tens of ps). This allowed us to minimize the effect of laser intensity fluctuations. Positive times correspond to the probe pulse (800 nm or $400 \mathrm{~nm}$ ) coming after the pump pulse (267 nm).

The ions are detected by the microchannel plate detector of a TOF-MS perpendicular to the molecular beam and the lasers. When working with $800 \mathrm{~nm}$ probe laser, the carrier gas was switched from Argon to Helium because of an intense signal from ionization of Argon.

The photoelectrons are detected in the opposite direction by a velocity map imaging spectrometer based on the design of Eppink and Parker ${ }^{38}$. The electrostatic optics is adjusted to accelerate the electrons perpendicular to the molecular beam in a velocity mapping electric field. The polarisations of the lasers are both set parallel to the plane of the detector. The photoelectron signal is detected by a position-sensitive imaging detector composed of microchannel plates coupled with a phosphor detector monitored by a charge-coupled device camera (LaVision). This system allows us to project the spherical distribution of the photoelectrons' speed (directly linked to their energy) on the planar surface of the camera detector. The treatment by Abel inversion of this projection with an adapted algorithm (Hansen \& Law) after symmetrisation of each image enables us to recreate the original spherical distribution and to extract information on the radial and angular energy distribution of the photoelectrons. This finally gives access to the energy distribution of the photoelectrons for each time step recorded. In the present conditions, the electron energy range observed was $0-3.4 \mathrm{eV}$ with an energy resolution of about $0.1 \mathrm{eV}$ (at $1 \mathrm{eV}$ ). Prior to each photoelectrons 
measurement, TOF mass spectra have been measured in order to check for the presence of trace compounds such as dimethylether (DME) and pyridine (used for internal calibration).

Data acquisition and analysis is achieved through home-made Labview-based software. Each photoelectron image and mass spectrum was averaged over 1000 laser shots per pump-probe delay. In each case, a baseline was calculated and subtracted. In the case of mass spectra, the baseline was calculated by averaging the signal before each peak of interest. This permits to correct the influence of baseline drifts due to strong signals occurring at smaller masses (for example the influence of the monomer signal on the clusters or dimers). In the case of the photoelectron images, an average image of the one-colour signal (negative times) was subtracted to all images within a series. The one-colour signal indeed presents features characteristic of the electronic states accessible by one-colour multiphoton absorption. The fitting procedure of the transients involves convolution of a multipleexponential decay by the laser cross-correlation. The zero-delay time and the laser crosscorrelation width are fixed parameters in the fitting procedure, and deduced from an independent in situ ionization measurement of DME or water in the molecular beam. The cross-correlation widths of the lasers (assumed to be Gaussian-shaped) obtained are of the order of $80 \mathrm{fs}(267 / 400 \mathrm{~nm})$ or $120 \mathrm{fs}(267 / 800 \mathrm{~nm})$. The relative precision of the values obtained for the lifetimes is estimated to be $\pm 10 \%$.

\section{Results and discussion}

\section{A. Bare 9-Methyladenine}

\section{Time-resolved photoionisation spectroscopy}

The time-dependent ion transients of 9MeAde measured at the parent ion mass channel for two different pump/probe schemes (267/400 vs. 267/800 nm) are shown in Figure 1b. The $267 / 400 \mathrm{~nm}$ pump-probe data, already published, ${ }^{27}$ will be used as a reference for the new sets of data reported in the present study. The two 9MeAde signals show very similar temporal behaviours, which can be reproduced by a two-component exponential decay previously assigned to the initially excited $\pi \pi^{*}\left(\mathrm{~L}_{\mathrm{b}}\right)$ and $n \pi^{*}$ state. A first ultrafast component of typically $100 \mathrm{fs}$ is followed by a slower step in the picosecond time range. The lifetimes $(95 / 1240 \mathrm{fs})$ are found to be the same within the measurement precision. 
Another relevant parameter in time-resolved measurements is the relative weight of the two components, which depends on the nature of the excited states (which controls the photoionisation efficiency) and can therefore provide information on the deactivation pathways. In the present case, we observe similar relative weights of the two components (see the heights of the calculated components in Figure 1) when comparing the two different pump/probe schemes, which suggests the absence of specific effect due to the probe process (400 vs $800 \mathrm{~nm}$ photons). In the following, this parameter will be used extensively to characterise the excited state dynamics.

At this stage of the analysis, it should be mentioned that, in pump-probe experiments at high laser intensities, the signals measured may be perturbed by large one-colour signals and pump-probe signals due to strong laser intensities, as well as significant fragmentation of the parent 9MeAde ions following the photoionisation probe. These phenomena may have significantly affected previous measurements and corresponding fits. ${ }^{25,26,28}$ In the present study, the laser intensities were kept as low as possible and the signal for negative delay times, corresponding roughly to one-colour background signals, was low ( $\sim 10-20 \%)$. This signal was found to be constant and equal to the signal at very long positive delays ( $>10 \mathrm{ps})$. This has been checked prior to each measurement in order to ensure appropriate and steady experimental conditions (moderate laser intensity and focusing, good spatial overlap of the lasers, controlled expansion conditions). Owing to the good signal-to-noise ratio (and our excellent time resolution) together with the minimized one-colour signal, an accurate fit of the parent ion transient based on a biexponential model was obtained without addition of a third very long-lived (ns) channel. Also, the absence of any multi-photon coherent absorption (laser cross-correlation), as already suggested, ${ }^{27}$ was confirmed.

Concerning the second perturbing effect, fragmentation is commonly observed in timeresolved experiments using photoionisation as a probe. Because of the large intensities of the probe lasers usually used, one cannot avoid it because of the large probability of ion photoexcitation during the probe laser pulse. The following analysis will nevertheless show that this phenomenon provides an interesting alternative way to monitor the excited state dynamics.

In the present experiment, the mass spectrum of 9MeAde shows the presence of many fragment ions ( $5-10 \%$ of the parent intensity) besides the intense parent peak. The most intense fragment ion (122 amu) may correspond to the opening of the six-member ring and loss of either $\mathrm{HC} 2 \mathrm{~N} 3$ or $\mathrm{N} 1 \mathrm{C} 2 \mathrm{H}(27 \mathrm{amu})$ (see Figure 1a for the numbering). As a single 
photon excitation at $267 \mathrm{~nm}(4.64 \mathrm{eV})$ cannot cause prompt dissociation in 9MeAde (it would require the breaking of two $\mathrm{C}-\mathrm{N}$ bonds), the fragmentation observed must have occurred in the ion after absorption of several probe $\left(400 \mathrm{~nm}\right.$ or $800 \mathrm{~nm}$ ) photons (total energy $>12 \mathrm{eV}^{39}$ ). It is noteworthy that the fragmentation in the ionic state may be relatively slow, on a timescale ranging from ps to tens of ns, depending upon the excess energy and the size of the molecular system. As the fragment ions have as precursor the parent ions formed during the probe pulse, their pump-probe time dependence is expected to be the same. Interestingly, the pump-probe time profile measured at the fragment mass (122 amu) clearly differs from that of the parent. The fragment decay signals (Fig. 1c) correspond essentially to the second component observed on the parent ion. It can be fitted by a rising exponential of 10-20 fs followed by a monoexponential decay almost identical to the second component value measured on the parent ion. This suggests that probing the dynamics from the fragment signal focuses essentially on the late part of the dynamics. It should be mentioned that this fragment signal is of course taken at the expense of the parent signal (namely here essentially the second component). The same type of observations have been previously reported on other smaller systems $^{36,37,40}$ It has been shown that an increased vibrational content in the excited PES of the molecule can lead, after ionization, to an enhancement of the fragment ion signals, therefore depleting the parent ion signal. Measuring differences between the temporal response of the parent and fragment ions can be a way to visualize the regions on the PES where the ionic fragmentation is favoured by vibrational excitations acquired in the excited neutral state. The comparison between the present parent and fragment ion transients nicely illustrates this effect on 9MeAde and brings evidence for the involvement of two excited electronic states with different vibrational contents.

These results demonstrate that the presence of fragmentation can significantly affect the temporal decay measured for the parent ion. The perturbation observed, i.e., the decrease of the second component's weight will be nicely confirmed by photoelectron spectroscopy (see next section). Of course, such a fragmentation effect is expected to be still more dramatic in the case of clusters, characterised by smaller binding energies. The above discussion explains why it may be difficult to compare the present results with previously published decays possibly acquired under different experimental conditions (strong fragmentation effects due to large laser intensities and/or presence of molecular clusters). Having in mind these limitations, one can nevertheless note the consistency of the present results with previous works at different excitation energies $(272,263 \text { and } 250 \mathrm{~nm})^{26}$ for Ade. In this former study 
all the transients show biexponential decays with no significant change of the first lifetime but an increase of the second one, from $\sim 2.3 \mathrm{ps}$ at $272 \mathrm{~nm}$ to $\sim 1$ ps at $250 \mathrm{~nm}$.

\section{Time-resolved photoelectron velocity imaging of 9 MeAde}

Photoelectron spectroscopy is sensitive to both the molecular orbital configurations and the vibrational content, and is therefore a technique of choice to apprehend the electronic relaxation processes in molecules. The electron velocity imaging technique used in the present work is moreover a very sensitive method because the electron counting rate may be very high and similar to that for the corresponding ions.

In a typical image (see Figure 2d), radial (electron energy) and angular (spatial anisotropy if any) dependences can be observed. The images can be integrated over all angles (thus disregarding any angular dependence) in order to focus on their time dependence. Temporal profiles of photoelectron intensities integrated over the whole kinetic energy range are shown in figure 1a. The temporal profiles of the mass-resolved and photoelectron measurements show the same lifetimes (within the fit precision). The much stronger second component in the photoelectron measurement compared to the parent photoion decay is qualitatively consistent with a large depletion of the second component due to fragmentation as discussed in the previous section. The qualitative agreement between the two lifetime measurements nevertheless supports the absence of perturbing trace compounds in the photoelectron experiments.

Time-resolved photoelectron kinetic energy distributions also provide spectral information on the electronic character of the excited states. ${ }^{25,41}$ The electron energy distributions corresponding to the two components (95 fs and $1240 \mathrm{fs}$ ) in the 267/400 nm excitation scheme are shown in Figure 2a. The energy distribution corresponding to the second component is easily extracted by integrating a time window where the first component has already decayed to zero ( $\sim 1$ ps after the zero time delay). In contrast, the energy distribution of the first component cannot be obtained directly. In this case, we have considered only one time channel, close to the zero delay, where the relative weight of the first component compared to the second is the highest, and have determined for different energy windows the weight of the first component at this delay in the dynamics. It is then possible to extract the first component contribution from the total energy distribution obtained at this specific delay. The bottom scale of Figure $2 \mathrm{a}$ shows the kinetic energy of the 
photoelectrons, whereas the upper scale gives the corresponding bonding energies of the electrons assuming a $267 / 2 \times 400 \mathrm{~nm}$ photoionisation scheme and a total energy of about 10.84 $\mathrm{eV}$. The two curves have different envelopes, characterised by a large energy spread and very slow increase of the photoelectron intensity (reading from high to low kinetic energies). The first component is related to electron energies ranging from 0.2 to $\sim 2.5 \mathrm{eV}$ while the onset of the second component distribution is shifted to lower kinetic energies by $\sim 0.5 \mathrm{eV}$ and shows a steep increase at $1.25 \mathrm{eV}$.

These results can be analysed based from the comparison of the experimental ionization thresholds with those expected from Koopman's ionization correlations ${ }^{42-44}$ combined to well characterised He (I) photoelectron spectra. The adiabatic ionization energy of jet cooled 9H-Ade has been determined by two-colour ionization spectroscopy to be 8.606 eV. ${ }^{19}$ By comparison of this value with $\mathrm{He}(\mathrm{I})$ photoelectron spectra of gas phase adenine and $9 \mathrm{MeAde},{ }^{45}$ we estimated the adiabatic ionization energy of jet-cooled $9 \mathrm{MeAde}$ to be $\mathrm{IE}_{0}=8.5$ $\mathrm{eV}(\pi$ hole $)$ and $\mathrm{IE}_{1}=9.5 \mathrm{eV}$ (n hole). When exciting at $267 \mathrm{~nm}(4.64 \mathrm{eV})$, the vibrational energy in the $\mathrm{L}_{\mathrm{b}} \pi \pi^{*}$ state of $9 \mathrm{MeAde}$ (origin transition at $4.48 \mathrm{eV}^{18}$ ) is of the order of 0.164 $\mathrm{eV}$ and slightly more $(\sim 0.24 \mathrm{eV})$ in the $n \pi^{*}$ state, if we assume that 9MeAde and $9 \mathrm{H}$-Ade follow a similar trend for the $n \pi *$ state $\left(\sim 600 \mathrm{~cm}^{-1}\right.$ red shift $) .{ }^{17}$ The Franck Condon principle implies (for vertical transitions) that most of the vibrational energy in the excited neutral states is transferred to the ion. The estimated electron energy at threshold after ionization of the $\pi \pi^{*}$ state to the $\mathrm{D}_{0}$ state is then $\sim 2.18 \mathrm{eV}(10.84-8.5-0.164)$. This value (red star in Figure $2 \mathrm{a}$ ) corresponds fairly well to the half maximum of the $\sim 95$ fs component curve. The estimated energy at threshold for the $n \pi^{*}$ state (blue star in fig.2a) is $\sim 1.1 \mathrm{eV}$ if we suppose that it preferentially ionizes to the $\mathrm{D}_{1}$ state (n hole). ${ }^{25}$ The step-like intensity increase in the experimental curve $(\sim 1.25 \mathrm{eV})$ corresponds fairly well to this value. The observed weak signal at threshold is located at higher energies $(\sim 2 \mathrm{eV})$ which may indicate that transitions from the $n \pi *$ state to the $\mathrm{D}_{0}$ continuum are also weakly allowed. This is probably due to small deviations from the simple Koopman's picture and the strong coupling between the two states. ${ }^{46,47}$ The very slow rise of the Franck Condon envelope found for both states (not a step function) may indicate different geometries of the neutral $\mathrm{S}_{1}, \mathrm{~S}_{2}$ and ionic $\mathrm{D}_{0,1,2}$ states of 9MeAde.

The temporal profiles for energy-selected photoelectron intensities (see Figure 2c) show all biexponential decays fitted with the same lifetimes as for the all-photoelectrons profiles. Different relative weights of the components are however found depending upon the 
kinetic energy selected: for example, the first component's weight is very small for electron energies in the 0.85-1.2 eV range, and increases for higher electron energies (1.9-2.5 eV). This observation is a direct consequence of the different energy distributions associated to the two components.

In a recent photoelectron study on Ade, an additional minor relaxation channel with a short lifetime (assigned to a $\pi \sigma^{*}$ state) was suggested to occur ${ }^{24,25}$ when exciting specifically at $267 \mathrm{~nm}$ (and not at $250 \mathrm{~nm}$ ). In that case, the envelope of the electron kinetic energy distribution of the first component showed an additional low-energy electron signal $(<0.7 \mathrm{eV})$ as compared to the present one. Indeed, a careful comparison of the time profiles obtained in this earlier study (integrated over all electron energies) with the present one (fig. 1a) indicates clearly that the relative weight of the first component observed for the $267 \mathrm{~nm}$ excitation was anomalously intense in this former study, while the components' ratio at $250 \mathrm{~nm}$ excitation is similar to the present photoelectron time profile, suggesting the absence of a marked dependence of the dynamics on the excitation wavelength. Such a feature would be in qualitative agreement with lifetimes measured at several wavelengths $(272,263$ and $250 \mathrm{~nm})$ in a previous series of photoion probe experiments (showing similar behaviour with excitation at 263 and $250 \mathrm{~nm}){ }^{26}$ A likely explanation for the additional low-energy photoelectron signal $^{25}$ is the accidental presence of trace compounds in the jet during these $267 \mathrm{~nm}$ measurements, for example water and Ade-water clusters, which are found to be very shortlived (see below). These species may have been much less populated or absent during the 250 nm experiment.

The present photoelectron results, namely the agreement of the kinetic energy distributions for the two temporal components with the expected vertical ionization potentials and the excellent fit of the energy-selected temporal profiles with biexponential decays with the same lifetimes, are consistent with the complementary photoion data, on both the parent and fragment ions, whatever the probe scheme used (400 vs. $800 \mathrm{~nm}$ ). They support the involvement of only two electronic states in the deactivation of 9MeAde after $267 \mathrm{~nm}$ excitation. The directly excited short-lived $\pi \pi^{*}$ electronic state ( $\sim 100$ fs component) relaxes to a $n \pi^{*}$ state (second component of $\sim 1200 \mathrm{fs}$ ), exhibiting a significant vibrational content. In addition, there is no evidence for the observation of a long-lived channel, involving a triplet state for instance (in contrast to the earlier photoelectron study ${ }^{25}$ ).

At higher energy $(250 \mathrm{~nm})$, photoelectron studies ${ }^{25}$ on the relaxation of Ade excited states show similar temporal profiles, suggesting the involvement of electronic states of the 
same nature, even if different $\pi \pi^{*}$ states are probably optically excited at this shorter wavelength $\left(\mathrm{L}_{\mathrm{b}}\right.$ and/or $\left.\mathrm{L}_{\mathrm{a}}\right)$.

\section{B. 9MeAde - water clusters}

The time-dependence of the 9MeAde hydrates is reflected by the transients profiles recorded in the 9 MeAde- $\mathrm{W}_{\mathrm{n}}(\mathrm{n}=0-10)$ mass channels (selected sizes are shown in Figure 3 ). The carrier gas used for the expansion was argon in the measurement with the $400 \mathrm{~nm}$ probe and helium with the $800 \mathrm{~nm}$ probe. A great care has been taken to prevent the formation of 9MeAde dimer (and higher clusters) and the corresponding hydrates. The molecular beam expansion conditions were optimised to produce narrow distributions of hydrates of the monomer only. The apparent cluster distribution vanishes for $\mathrm{n}>10$.

All transients are fitted by a biexponential decay ( 100/ 1000 fs), with a much weaker second component as compared to the monomer measured under dry conditions (Figs. 1 and 3). The lifetimes of the first component is found to decrease significantly with the cluster size, from $120 \mathrm{fs}(110 \mathrm{fs})$ for $\mathrm{n}=1$ down to $100 \mathrm{fs}(80 \mathrm{fs})$ for $\mathrm{n}=6$ for example when probed by the $267 / 400 \mathrm{~nm}(267 / 800)$ pump-probe schemes. The same trend is observed for the lifetimes of the second component, going from $980 \mathrm{fs}(1000 \mathrm{fs})$ to $750 \mathrm{fs}(500 \mathrm{fs})$, and its relative weights.

These results, in qualitative agreement with earlier studies, ${ }^{28,48}$ show an apparent drastic change of the 9MeAde dynamics in a water cluster environment, in particular, a dramatic decrease of the second component weight compared to the isolated molecule. This new temporal features in clusters can have two possible origins. On one hand, it can be due to a specific solvent effect on the electronic dynamics of the 9MeAde molecule. On the other hand, it could be a consequence of an intense ion fragmentation, as described above for the isolated monomers, but much more important in these species because of their lower binding energy. Dissociation in the neutral excited state is energetically possible but its effect are probably small for two reasons: i) first, the excess energy in the excited state does not allow to evaporate more than a few water molecules, since the corresponding cooling slows down the following evaporation events, ii) second, evaporation is probably negligible within the 1 ps timescale already for small size hydrates $(n>3) .{ }^{49}$

Evidence for an intense fragmentation in the ionic state can be found in the temporal behaviour of the ion signal measured in the $9 \mathrm{MeAde}^{+}$mass channel (Figure 3). As compared to the isolated monomer (Fig.1b), the monomer transient with the $267 / 400 \mathrm{~nm}$ pump/probe 
scheme shows a longer first component and an apparently weaker second component. For comparison, the decay of the $9 \mathrm{MeAde}-\mathrm{W}_{1}$ complex is shown on the same figure. The apparent greater lifetime of the first component (140 fs) may be explained by contributions of cluster fragment signals, which reach a maximum value after a few tens of fs delay as compared to their parent. The smaller weight of the second component of this signal resulting from the sum of the isolated monomer and fragmented hydrates suggests that the relative weight of the second component in the intrinsic dynamics of the hydrates is small. This observation is further supported by the experiment with the $800 \mathrm{~nm}$ probe carried out with $\mathrm{He}$ as carrier gas. In this case, a strong deviation electric field, perpendicular to the molecular jet, has to be used in the mass spectrometer flight tube in order to detect essentially higher mass species. Because of the larger kinetic energy in He than in Ar, this effect is here very selective. The temporal signal recorded in the $9 \mathrm{MeAde}^{+}$mass channel is very similar to that of the clusters in terms of components relative weight, which suggests in this case a negligible contribution of isolated parent monomers to the signal. Moreover, this signal is shifted by $\sim 20$ fs to longer times, as compared to the clusters transients' rise time (see insert in Figure 3). This observation ensures that, because of the electric deviation in the TOF mass spectrometer chosen to detect preferentially clusters, the signal in the monomer mass channel recorded in He jet with $267 / 800 \mathrm{~nm}$ pump-probe scheme is essentially due to fragmented ionized hydrates and contains the dynamical contributions of a whole range of cluster sizes.

Under these very specific experimental conditions, the weakness of the second component in the monomer channel ensures both the existence and the weakness of this component in the clusters. It demonstrates the intrinsic character of the cluster dynamics observed and its qualitatively different nature as compared to the isolated monomer. From the comparison of the temporal shapes of fragment monomer and $9 \mathrm{MeAde}^{+}-\mathrm{W}_{\mathrm{n}}$, it is clear that a significant change in terms of components relative weight is operative as soon as one water molecule is bound to 9MeAde. The existence of only two preferential hydration sites on the 9methylated molecule, i.e., with the water molecule bridging the amino group and either the $\mathrm{N} 1$ or the N7 atom, ${ }^{50-54}$ therefore suggests that the effect of the most strongly bonded solvent molecule is similar in these two sites. The progressive weak change in the dynamics lifetime with the cluster size can be simply understood by a progressive solvation of the nucleobase from these sites.

The present cluster study can be compared to two previous gas phase works on hydrated adenine. ${ }^{28,48}$ In contrast to the present results, both studies reported monoexponential decays. In the first study, the similar lifetimes found for all the clusters ( $\sim 220 \mathrm{fs})$ may be 
easily explained by the low temporal resolution (400 fs) in this experiment. ${ }^{48}$ The results of the second study (60 - 80 fs and 110 fs for 263 and $250 \mathrm{~nm}$ excitations, respectively) suffered from strong laser intensities and therefore from a very intense fragmentation of the ionized Ade- $\mathrm{W}_{\mathrm{n}}$ clusters. This caused a loss of the second component in the cluster mass channels, ${ }^{28}$ which should have been found in the monomer channel, but could not be distinguished from the intense second component of the unsolvated 9MeAde monomer which was detected in this experiment (as in the present measurement in the Ar jet).

Finally, the present results on the 9MeAde-water clusters can be compared with lifetimes of 9MeAde related molecules (Ado, dAdo, AMP, dAMP) measured in aqueous solution. Double exponential decays with a weak second component have been observed by fluorescence up-conversion ${ }^{30}$ on dAdo and dAMP. These results (in particular the lifetimes of $100 / 420$ fs and 100/520 fs, respectively), are in excellent agreement with our cluster measurements. Other studies on these systems, using both fluorescence up-conversion and transient absorption techniques, also reported sub-ps signals, although fitted to monoexponential decays with lifetimes ranging from 0.22 to 0.53 ps. $^{6,29,55}$ The differences between the fluorescence results could be related to differences in the experimental conditions (temporal resolution, sensitivity). The different results obtained by transient absorption cannot be easily reconciled with fluorescence measurement and the absence of a second component in transient absorption measurements is not understood at present. In spite of this difference, the comparison of the results from both solution studies and the present water cluster approach show clearly great similarities, namely similar subpicosecond lifetimes for the radiationless relaxation pathways.

\section{Comparison between experimental results and theoretical models}

Several recent theoretical papers ${ }^{13-16,33-35}$ provide a convenient theoretical framework to interpret the ultrafast deactivation pathways of adenine.

The energetics of the excited states, and the crossings between them or with the ground state, via conical intersections $(\mathrm{CI})$, where radiationless transitions can take place, have been calculated as well as the various barriers along the deactivation pathways. On the basis of these results a qualitative picture can be drawn (Figure 4).

Vertical excitation of the isolated 9 MeAde populates the lowest excited state ${ }^{1} \mathrm{~L}_{\mathrm{b}}$ far from its equilibrium region, causing the initial displacement of the wave packet towards the ${ }^{1} \mathrm{~L}_{b} \pi \pi *$ 
local minimum, labelled $\left({ }^{1} \mathrm{~L}_{\mathrm{b}}\right)_{\text {Min. }}$. Calculations show the presence of one or two paths for the decay of this $\left({ }^{1} \mathrm{~L}_{\mathrm{b}}\right)_{\text {Min }}$ relaxed geometry with a small or virtually inexistent barrier to the $\left(\mathrm{n} \pi^{*}\right)_{\text {Min }}$ (via the $\left(\mathrm{L}_{\mathrm{b}} / \mathrm{n} \pi^{*}\right)_{\mathrm{X}} \mathrm{CI}^{13,14,33-35}$ ) or to the $\mathrm{L}_{\mathrm{a}}$ state (via the $\left({ }^{1} \mathrm{~L}_{\mathrm{a}} / \mathrm{L}_{\mathrm{b}}\right)_{\mathrm{X}} \mathrm{CI}^{35}$ ) respectively. The competition (branching) between these decay channels may depend on the excess excitation energy. ${ }^{14,35}$ Upon excitation of the ${ }^{1} \mathrm{~L}_{\mathrm{a}}$ state, there is a barrierless pathway from the FC geometry to the conical intersection of ${ }^{1} \mathrm{~L}_{\mathrm{a}}$ with the ground state, labelled $\left({ }^{1} \mathrm{~L}_{\mathrm{a}}\right)_{\mathrm{X}}{ }^{35}$ The state can therefore either decay straight to the ground state, or induce population of the ${ }^{1} \mathrm{~L}_{b}$ state and then of the $n \pi^{*}$ state when passing through the corresponding $\left(\mathrm{L}_{\mathrm{a}} / \mathrm{L}_{\mathrm{b}}\right)_{\mathrm{X}}$ and $\left(\mathrm{L}_{\mathrm{a}} /\right.$ $\left.\mathrm{n} \pi^{*}\right)_{\mathrm{X}} \mathrm{CI}$, which are found to be peak-shaped. ${ }^{56}$ In contrast, the decay paths of the $\left(\mathrm{n} \pi^{*}\right)_{\text {Min }}$ through the conical intersections $\left({ }^{1} \mathrm{~L}_{\mathrm{a}}\right)_{\mathrm{X}}$ and $\left(\mathrm{n} \pi^{*}\right)_{\mathrm{X}}$ with the ground state have sizable barriers $(0.1 \mathrm{eV}$ or more $){ }^{35}$ The conical intersections involved in these pathways are characterised by strongly bent geometries, and are accessed through out-of-plane deformation modes of the six-membered ${ }^{13,14,33-35}$ ring. The presence of a specific relaxation channels involving $\pi \sigma^{*}$ states (repulsive along the $\mathrm{N} 9-\mathrm{H}$ bond, for example) has been repeatedly suggested in theoretical works. These news channels have however been recently calculated to open up at higher energy (230-220 nm), ${ }^{14-16}$ due to the existence of a high barrier $(\sim 1 \mathrm{eV})$. Calculations also suggest that from the $\left(\mathrm{n} \pi^{*}\right)_{\text {Min }} \mathrm{a}^{3}\left(\pi \pi^{*}\right)$ triplet state can be populated by intersystem crossing. This channel has not been observed experimentally either in the isolated or in the water cluster conditions which is in agreement with the very low ISC quantum yield measured for dAdo in water solution $\left(<10^{-3}\right)^{57}$.

The mass-resolved and photoelectron experimental data on the photophysics of the canonical tautomer of adenine (or 9MeAde) support the theoretical models proposed (qualitative scheme Figure 4). The excited 9MeAde molecule shows an ultrafast decay of the initially excited $\pi \pi^{*} \mathrm{~L}_{\mathrm{b}}$ state (lifetime $\tau_{1} \sim 100 \mathrm{fs}$ ) to the $\mathrm{n} \pi^{*}$ state. The involvement of out-ofplane vibrational modes was experimentally evidenced ${ }^{27}$ by a longer time-scale dynamics ( $\sim 200 / 3000 \mathrm{fs}$ ) measured in dimethyladenine, related to the lower frequency of the amino group inversion upon methylation. The $n \pi^{*}$ state then decays to the ground state with a $\tau_{2}$ lifetime ranging from $\sim 10-50 \mathrm{ps}$ (near threshold) to $1.25 \mathrm{ps}$ (267 $\mathrm{nm}$ excitation).

The short progression of vibronic lines extending only over $800 \mathrm{~cm}^{-1}$ in the gas phase UV spectrum together with the lifetime dependence on the excitation energy are in qualitative agreement with the existence of a low barrier, which is easily crossed when exciting at UV wavelength below $267 \mathrm{~nm}$. The large signal observed for the $n \pi^{*}$ state indicates that this is the dominant relaxation channel and that other decay channels play only a minor role. At high 
excitation energy $(250 \mathrm{~nm})$, the relative amplitude of the two components changes weakly, which might be the sign of the involvement of the $\mathrm{L}_{\mathrm{a}}$ state in the dynamics.

In the case of the hydrates, our very detailed experimental results provide enough data to propose a new pathway for the relaxation of their excited states, based on theoretical predictions combined to general considerations about hydration effect (Fig. 4, lower scheme). The excited 9MeAde in a water cluster environment shows indeed a dramatically different temporal behaviour, in particular a very weak second $n \pi^{*}$ component is observed which may indicate that a new relaxation channel is operating for the initially excited $\pi \pi^{*}$ state. This behaviour is probably related to the reordering of the states as expected for these molecules in polar solvents. The ${ }^{1} \mathrm{~L}_{\mathrm{a}}$ state is the most polar state among the lowest singlet states (dipole moment $4.7 \mathrm{D}^{16}$ ). This state (and to a lesser extend ${ }^{1} \mathrm{~L}_{\mathrm{b}}$ ) is thus expected to be stabilised (redshifted) in polar solvents. The opposite is expected for the less polar (relative to the ground state) $n \pi^{*}$ state which will be blue-shifted. The destabilisation of the $n \pi^{*}$ state may induce a

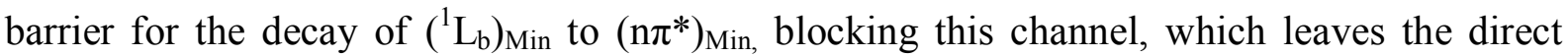
decay of $\left({ }^{1} \mathrm{~L}_{\mathrm{b}}\right)_{\text {Min }}$ through $\left({ }^{1} \mathrm{~L}_{\mathrm{a}}\right)_{\mathrm{X}}$ as the dominant one. On the other hand, the most stabilised ${ }^{1} \mathrm{~L}_{\mathrm{a}}$ state may be accessible at $267 \mathrm{~nm}$ excitation, and may decay in a barrierless manner to the the ground state via the $\left({ }^{1} \mathrm{~L}_{\mathrm{a}}\right)_{\mathrm{X}} \mathrm{CI}$ and/or populate the $\left({ }^{1} \mathrm{~L}_{\mathrm{b}}\right)_{\text {Min }}$ and $\left(\mathrm{n} \pi^{*}\right)_{\text {Min }}$ through the peaked conical intersections ${ }^{55}\left(\mathrm{~L}_{\mathrm{a}} / \mathrm{L}_{\mathrm{b}}\right)_{\mathrm{X}}$ and $\left(\mathrm{L}_{\mathrm{a}} / \mathrm{n} \pi^{*}\right)_{\mathrm{X}}$, respectively. When exciting at $267 \mathrm{~nm}$, the biexponential transient signal of the hydrates may thus contain the contributions of the $\mathrm{L}_{\mathrm{a}}$ and $\mathrm{L}_{\mathrm{b}}$ excited states which can not be experimentally distinguished. Moreover, the weak $n \pi *$ amplitude could reflect a competition between two different decay channels for the $\mathrm{L}_{\mathrm{b}}\left(\right.$ or $\mathrm{L}_{\mathrm{a}}$ ) $\pi \pi^{*}$ state, namely i) formation of the $n \pi^{*}$ intermediate or ii) direct decay to the ground state.

Finally, the solvation stabilised $\pi \sigma^{*}$ state might also play a role in the dynamics of hydrated species, as suggested in a recent study on adenine-water complexes dynamics, for excitation at 267 and $250 \mathrm{~nm}^{28}$ However, such a relaxation pathway is expected to be negligible in the hydrates' dynamics, since no signal corresponding to (9MeAde $-\mathrm{H}$ ) species (hydrated or not) could be detected in our mass-resolved experiments in presence of water.

\section{Conclusion}

The present dynamics investigation allows a more refined description of the photophysics of the 9MeAde in isolated and microhydrated environment near the region of fluorescent-to-dark state switching. The combined use of two complementary detection 
techniques, namely time-resolved ion mass spectrometry, on both the parent and fragments, and time-resolved photoelectron imaging, provides a unique tool to probe the detailed characteristics (nature, lifetime...) of the excited states involved in the relaxation. Based upon our results and previously reported studies, the following model for internal conversion pathways in this purine base is proposed.

In the isolated molecule, excitation at $267 \mathrm{~nm}$ populates the bright $\pi \pi^{*} \mathrm{~L}_{\mathrm{b}}$ state. The present results indicate that this state decays rapidly ( $\sim 100 \mathrm{fs})$ to the $n \pi^{*}$ state which has a lifetime of about 1 ps. This is evidenced by i) the specific photoelectron energy distributions of these states, or ii) the different fragmentation efficiencies following their photoionisation, illustrating their different vibrational contents. Excitation at $250 \mathrm{~nm}$ leads to a similar temporal behaviour, suggesting an initial excitation of the $\mathrm{L}_{\mathrm{a}}$ state followed by internal conversion to the $\mathrm{L}_{b}$ and $n \pi *$ states. Finally, following $267 \mathrm{~nm}$ excitation, no other channel involving for instance a $\pi \sigma^{*}$ state or a long-lived (triplet) state could be detected in the present photoelectron study.

In the microhydrated molecule, our measurements proved the existence of a biexponential dynamics, that was only observable thanks to carefully controlled experimental conditions and a specific ion collection optimised for fragment detection. The temporal behaviour of the hydrates is dominated by a very strong ultrafast $(\sim 100 \mathrm{fs})$ first component, followed by a minor longer second component. This intrinsic dynamics of the hydrates is discussed based on the expected solvent effects. The interaction with water will stabilise the $\mathrm{L}_{\mathrm{a}}$ and $\mathrm{L}_{\mathrm{b}} \pi \pi^{*}$ states according to their polarisability, suggesting that both states are excited simultaneously at $267 \mathrm{~nm}$ in the hydrates. Finally, the study of the 9MeAde - water clusters shows that the study in a supersonic expansion combined to time-resolved mass spectrometry enables to document the gradual effects of solvation on the photophysics of a biomolecule, with a sensitivity which may be better than that achieved in condensed phase studies.

The models for the deactivation mechanism in the pyrimidine nucleobases, uracil and cytosine, as well as their relevant derivatives and hydrates are currently being investigated using the comprehensive time-, energy- and mass-resolved spectroscopic approach.

\section{Acknowledgements}

The authors are happy to thank Drs. B. Soep, L. Poisson, for making available to us their photoelectron imaging spectrometer and their help in data processing, as well as $\mathrm{O}$. Gobert, the late P. Meynadier, M. Perdrix, F. Lepetit, J.F. Hergott, and D.Garzella, who are 
responsible for the developing, maintaining, and running of the femtosecond laser facility LUCA (Laser Ultra-Court Accordable) of the CEA, DSM/DRECAM at Saclay.

\section{Figure captions}

Figure 1. Time-resolved photoion and photoelectron transients observed (red dots) in 9MeAde with two pump-probe schemes $(267 / 400 \mathrm{~nm}$ and 267/800 nm).

a) Decays measured by a photoelectron imaging technique integrated over all electron energies, b) Decays measured by photoionisation with detection in the parent mass channel, c) Decays measured by photoionisation with detection in the $\mathrm{m}=122 \mathrm{amu}$ fragment channel. Orange and green curves show the convolution of the Gaussian cross-correlation with the first and second exponential decay components respectively. The sum of these two components with a small offset (accounting for one-colour signal) gives the blue curve fitting the experimental data.

Figure2. Time-resolved photoelectron spectroscopy of 9MeAde using the velocity imaging technique.

a) Energy distributions of the 9MeAde photoelectrons measured with the $267 / 400 \mathrm{~nm}$ excitation scheme, corresponding to the first (red) and second (blue) components of the decay (the red line displays a smoothing of the data). The two stars indicate the expected ionisation thresholds for the $\mathrm{D}_{0}$ (red) and $\mathrm{D}_{1}$ (blue) ion states. b) Typical photoelectron velocity imaging spectrum obtained after treatment of the raw data. c) Transients observed by integration of selected electron energies, and calculated first (orange) and second (green) components of the fitting curve. d) Example of a raw image, taken at zero time delay, and displaying the photoelectron intensity as a function of the position on the detector (increasing intensity colour scale from blue to red).

Figure 3. Time-resolved photoionisation of 9MeAde - water clusters displayed for two excitation schemes $(267 / 400$ and $267 / 800 \mathrm{~nm})$ 
Upper panel) The parent mass channel signal (red dots) is compared to the 9MeAde$\mathrm{H}_{2} \mathrm{O}$ cluster (black line). Insert reveals a $20 \mathrm{fs}$ shift between the maxima of the two curves in the case of $267 / 800 \mathrm{~nm}$ pump-probe scheme. Following panels) Transients for the 9 MeAde- $\left(\mathrm{H}_{2} \mathrm{O}\right)_{n}, \mathrm{n}=1,3$ and 5. In all cases, the fitting curves are displayed in blue, and the orange and green curves show the two components of the fit.

Figure 4. Two-dimensional schematic representation of the multidimensional potential energy surface featuring the excited states involved in the electronic relaxation of isolated 9MeAde and 9MeAde hydrates. Conical intersections ${ }^{13,14,33-35}$ are identified according to the notation (..) $)_{\mathrm{X}}$ by Blancafort. ${ }^{35}$ In the isolated base (upper scheme), $\mathrm{L}_{\mathrm{a}}$ excitation may populate the $\mathrm{L}_{\mathrm{b}}$ and $n \pi^{*}$ states. Two relaxation pathways are accessible from both $\mathrm{L}_{\mathrm{b}}$ and $\mathrm{n} \pi^{*}$ minima (noted (..) Min $_{\text {) }}$. The $\mathrm{L}_{\mathrm{b}}$ state relaxes mostly to $\mathrm{n} \pi^{*}$ state, and possibly to $L_{a}$ state, through small $(<0.1 \mathrm{eV})$ barriers. The relaxation of the $n \pi^{*}$ state (directly to the ground state or through the $\mathrm{L}_{\mathrm{a}}$ state) involves higher barriers, explaining a longer lifetime. In the hydrates (lower scheme), both $\mathrm{L}_{\mathrm{b}}$ and $\mathrm{L}_{\mathrm{a}}$ states may be excited. The opening of a direct relaxation path from $L_{a}$ to the ground state and the increased importance of the relaxation pathway from $L_{b}$ to $L_{a}$ state (minor in the isolated base) explain the dominant ultrafast first component observed, while the remaining $\mathrm{L}_{b}$ to $n \pi^{*}$ state path (now minor) can account for the very weak second component detected. of America 94 (1), 11 (1997).

A. R. Young, British Journal of Clinical Practice, 10 (1997).

H. Mukhtar and C. A. Elmets, Photochemistry and Photobiology 63 (4), 355 (1996).

R. N. Saladi and A. N. Persaud, Drugs of Today 41 (1), 37 (2005).

P. R. Callis, Annual Review of Physical Chemistry 34, 329 (1983). 
C. E. Crespo-Hernandez, B. Cohen, P. M. Hare, and B. Kohler, Chemical Reviews 104 (4), 1977 (2004).

M. Daniels and Hauswirt.W, Science 171 (3972), 675 (1971).

A. Reuther, H. Iglev, R. Laenen, and A. Laubereau, Chemical Physics Letters 325 (4), $360(2000)$.

A. Broo and A. Holmen, Journal of Physical Chemistry A 101 (19), 3589 (1997).

M. P. Fulscher, L. SerranoAndres, and B. O. Roos, Journal of the American Chemical Society 119 (26), 6168 (1997).

B. Mennucci, A. Toniolo, and J. Tomasi, Journal of Physical Chemistry A 105 (19), 4749 (2001).

C. Marian, D. Nolting, and R. Weinkauf, Physical Chemistry Chemical Physics 7 (18), 3306 (2005).

A. L. Sobolewski and W. Domcke, European Physical Journal D 20 (3), 369 (2002).

C. M. Marian, Journal of Chemical Physics 122 (10), 104314 (2005).

S. Perun, A. L. Sobolewski, and W. Domcke, Journal of the American Chemical Society 127 (17), 6257 (2005).

S. Perun, A. L. Sobolewski, and W. Domcke, Chemical Physics 313 (1-3), 107 (2005).

N. J. Kim, G. Jeong, Y. S. Kim, J. Sung, S. K. Kim, and Y. D. Park, Journal of Chemical Physics 113 (22), 10051 (2000).

D. C. Luhrs, J. Viallon, and I. Fischer, Physical Chemistry Chemical Physics 3 (10), 1827 (2001).

C. Plutzer and K. Kleinermanns, Physical Chemistry Chemical Physics 4 (20), 4877 (2002). (3), 317 (2002). 
N. J. Kim, H. Kang, Y. D. Park, and S. K. Kim, Physical Chemistry Chemical Physics 6 (10), $2802(2004)$.

I. Hunig, C. Plutzer, K. A. Seefeld, D. Lowenich, M. Nispel, and K. Kleinermanns, Chemphyschem 5 (9), 1427 (2004).

H. Kang, B. Jung, and S. K. Kim, Journal of Chemical Physics 118 (15), 6717 (2003).

S. Ullrich, T. Schultz, M. Z. Zgierski, and A. Stolow, Journal of the American Chemical Society 126 (8), 2262 (2004).

S. Ullrich, T. Schultz, M. Z. Zgierski, and A. Stolow, Physical Chemistry Chemical Physics 6 (10), 2796 (2004).

E. Samoylova, H. Lippert, S. Ullrich, I. V. Hertel, W. Radloff, and T. Schultz, Journal of the American Chemical Society 127 (6), 1782 (2005). 
H. W. Jochims, M. Schwell, H. Baumgartel, and S. Leach, Chemical Physics 314 (13), 263 (2005).

J. M. Mestdagh, J. P. Visticot, M. Elhanine, and B. Soep, Journal of Chemical Physics 113 (1), 237 (2000).

W. Fuss, W. E. Schmid, and S. A. Trushin, Journal of Chemical Physics 112 (19), 8347 (2000).

W. Fuss, W. E. Schmid, and S. A. Trushin, Journal of the American Chemical Society 123 (29), 7101 (2001).

P. Asselin, F. Piuzzi, J. Lecalve, M. Mons, and I. Dimicoli, Chemical Physics 181 (12), 271 (1994). 
A. K. Chandra, M. T. Nguyen, T. Uchimaru, and T. Zeegers-Huyskens, Journal of Physical Chemistry A 103 (44), 8853 (1999).

O. S. Sukhanov, O. V. Shishkin, L. Gorb, Y. Podolyan, and J. Leszczynski, Journal of Physical Chemistry B 107 (12), 2846 (2003).

M. Hanus, M. Kabelac, J. Rejnek, F. Ryjacek, and P. Hobza, Journal of Physical Chemistry B 108 (6), 2087 (2004).

J. Peon and A. H. Zewail, Chemical Physics Letters 348 (3-4), 255 (2001).

K. H. Kraemer, Proceedings of the National Academy of Sciences of the United States of America 94 (1), 11 (1997).

A. R. Young, British Journal of Clinical Practice, 10 (1997). 104 (4), 1977 (2004). M. Daniels and Hauswirt.W, Science 171 (3972), 675 (1971). A. Reuther, H. Iglev, R. Laenen, and A. Laubereau, Chemical Physics Letters 325 (4), $360(2000)$. A. Broo and A. Holmen, Journal of Physical Chemistry A 101 (19), 3589 (1997). 
M. P. Fulscher, L. SerranoAndres, and B. O. Roos, Journal of the American Chemical Society 119 (26), 6168 (1997).

B. Mennucci, A. Toniolo, and J. Tomasi, Journal of Physical Chemistry A 105 (19), 4749 (2001).

C. Marian, D. Nolting, and R. Weinkauf, Physical Chemistry Chemical Physics 7 (18), 3306 (2005).

A. L. Sobolewski and W. Domcke, European Physical Journal D 20 (3), 369 (2002).

C. M. Marian, Journal of Chemical Physics 122 (10), 104314 (2005).

S. Perun, A. L. Sobolewski, and W. Domcke, Journal of the American Chemical Society 127 (17), 6257 (2005).

S. Perun, A. L. Sobolewski, and W. Domcke, Chemical Physics 313 (1-3), 107 (2005). N. J. Kim, G. Jeong, Y. S. Kim, J. Sung, S. K. Kim, and Y. D. Park, Journal of Chemical Physics 113 (22), 10051 (2000).

D. C. Luhrs, J. Viallon, and I. Fischer, Physical Chemistry Chemical Physics 3 (10), 1827 (2001).

C. Plutzer and K. Kleinermanns, Physical Chemistry Chemical Physics 4 (20), 4877 (2002).

E. Nir, C. Plutzer, K. Kleinermanns, and M. de Vries, European Physical Journal D 20 (3), 317 (2002).

N. J. Kim, H. Kang, Y. D. Park, and S. K. Kim, Physical Chemistry Chemical Physics 6 (10), 2802 (2004).

I. Hunig, C. Plutzer, K. A. Seefeld, D. Lowenich, M. Nispel, and K. Kleinermanns, Chemphyschem 5 (9), 1427 (2004).

H. Kang, B. Jung, and S. K. Kim, Journal of Chemical Physics 118 (15), 6717 (2003). 
S. Ullrich, T. Schultz, M. Z. Zgierski, and A. Stolow, Journal of the American Chemical Society 126 (8), 2262 (2004).

S. Ullrich, T. Schultz, M. Z. Zgierski, and A. Stolow, Physical Chemistry Chemical Physics 6 (10), 2796 (2004).

E. Samoylova, H. Lippert, S. Ullrich, I. V. Hertel, W. Radloff, and T. Schultz, Journal of the American Chemical Society 127 (6), 1782 (2005).

C. Canuel, M. Mons, F. Piuzzi, B. Tardivel, I. Dimicoli, and M. Elhanine, Journal of Chemical Physics 122 (7), 074316 (2005).

H. H. Ritze, H. Lippert, E. Samoylova, V. R. Smith, I. V. Hertel, W. Radloff, and T. Schultz, Journal of Chemical Physics 122 (22), 224320 (2005).

T. Pancur, N. K. Schwalb, F. Renth, and F. Temps, Chemical Physics 313 (1-3), 199 (2005).

A. Eppink and D. H. Parker, Review of Scientific Instruments 68 (9), 3477 (1997). 
H. W. Jochims, M. Schwell, H. Baumgartel, and S. Leach, Chemical Physics 314 (13), 263 (2005).

J. M. Mestdagh, J. P. Visticot, M. Elhanine, and B. Soep, Journal of Chemical Physics 113 (1), 237 (2000).

P. Asselin, F. Piuzzi, J. Lecalve, M. Mons, and I. Dimicoli, Chemical Physics 181 (12), 271 (1994).

C. A. de Lange, edited by I. Powis, T. Baer, and C. Y. Ng (Wiley, New York, 1995), pp. 195.

J. Berkowitz, Photoabsorption, Photoionization, and Photoelecton Spectroscopy. (Academic, New York, 1979).

S. T. Pratt, P. M. Dehmer, and J. L. Dehmer, Journal of Chemical Physics 81, 3444 (1984).

J. Lin, C. Yu, S. Peng, I. Akiyama, K. Li, L. K. Lee, and P. R. LeBreton, Journal of the American Chemical Society 102, 4627 (1980).

A. Stolow, A. E. Bragg, and D. M. Neumark, Chemical Reviews 104, 1719 (2004).

C. P. Schick, S. D. Carpenter, and P. M. Weber, Journal of Physical Chemistry A 103 (49), 10470 (1999).

N. J. Kim, Y. S. Kim, G. Jeong, T. K. Ahn, and S. K. Kim, International Journal of Mass Spectrometry 219 (1), 11 (2002).

G. Gregoire, M. Mons, I. Dimicoli, C. Dedonder-Lardeux, C. Jouvet, S. Martrenchard, and D. Solgadi, Journal of Chemical Physics 112 (20), 8794 (2000).

J. D. Gu and J. Leszczynski, Journal of Physical Chemistry A 103 (15), 2744 (1999).

H. T. Kim, Journal of Molecular Structure-Theochem 673 (1-3), 121 (2004).

A. K. Chandra, M. T. Nguyen, T. Uchimaru, and T. Zeegers-Huyskens, Journal of Physical Chemistry A 103 (44), 8853 (1999). 
O. S. Sukhanov, O. V. Shishkin, L. Gorb, Y. Podolyan, and J. Leszczynski, Journal of Physical Chemistry B 107 (12), 2846 (2003).

54 M. Hanus, M. Kabelac, J. Rejnek, F. Ryjacek, and P. Hobza, Journal of Physical Chemistry B 108 (6), 2087 (2004).

J. Peon and A. H. Zewail, Chemical Physics Letters 348 (3-4), 255 (2001).

56 G. J. Atchity, S. S. Xantheas, and K. Ruedenberg, Journal of Chemical Physics 95 (3), 1862 (1991).

57 J. Cadet, W. Roth, and I. Fischer, edited by H. Morrison (John Wiley \& Sons, New York, 1990), Vol. 1, pp. 1. 